\title{
EL USO DE LA TECNOLOGÍA DE LA INFORMACIÓN Y LA COMUNICACIÓN Y EL DISEÑO CURRICULAR
}

\author{
Gisselle Gómez Ávalos \\ Estudiante de la Maestría en Planificación Curricular \\ Universidad de Costa Rica \\ San José, Costa Rica
}

Recibido: 27-XI-2007 • Aceptado 11-III-2008 • Corregido 26-IV-2008

\begin{abstract}
Resumen: En las sociedades actuales las tecnologías de la información y la comunicación impregnan todos los quehaceres de la vida de las personas, de ahí la importancia que cobran los procesos de alfabetización tanto informacional como digitales que provea de las herramientas necesarias tanto para el procesamiento de la información como para el manejo técnico de las mismas, lo que lleva a plantear un modelo educativo que contemple las diferentes dimensiones: la tecnológica, la pedagógica y la administrativa en los procesos de formación. De ahí que este artículo propone diferentes formas de implementarlas en el campo educativo que enriquezca los procesos de enseñanza-aprendizaje, introduciendo estrategias novedosas para aprender a interactuar con la tecnología a partir de una adecuada planificación curricular.
\end{abstract}

Palabras clave: Tecnología de la información y la comunicación, alfabetización digital e informacional, educación, currículo.

\section{Introducción}

El artículo pretende dar un aporte en el campo de la gestión curricular planteando una propuesta, que permita, al usuario de las Tic's, (Tecnologías de la información y la comunicación) expuesto al proceso de alfabetización, tanto informacional como digital, hacer manejo de ellas en el campo educativo, considerando las diversas dimensiones que están presentes cuando van a implementar: la tecnológica, la pedagógica y la administrativa. Esto lleva a replantear la labor educativa desde una perspectiva de construcción del conocimiento, que supere el enfoque de repetición de conocimientos, tanto por parte de los docentes como del estudiante, al aplicar estrategias de enseñanza-aprendizaje innovadoras que propicien más bien la construcción del saber.

Diversos estudios realizados en sitios como España, Argentina, México sobre la aplicación de las Tic's en el campo educativo, destacan la necesidad de incorporarlas, tanto en el ámbito secundario, como universitario, desde perspectivas que superen el manejo básico de la herramienta y que 


\begin{abstract}
In the current societies the technologies of information and communication impregnate all the tasks of people's life, from there the importance that is given to the processes of computing as well as digital alphabetization that provides the necessary tools for the information processing as well as the technical handling of them, which leads to raise an educative model that contemplates the different dimensions: the technological, the pedagogical and the administrative one in the formation processes. For that reason this study proposes different ways for implementing them in the educative field in a manner that enriches the education-learning processes, introducing novel strategies to learn interacting with the technology from an adequate curricular planning.
\end{abstract}

Key words: Technology of information and communication, digital and informational alphabetization, education, curriculum. trascienda la visión tradicional de la educación.

Esta revisión de estudios, Ariza y otros (s.f.); Chiecher y otros (2006); Martínez, y otros (2001; 2005; 2006); Dibut y otros (2006); López y otros (2006); Fazeli y otros (2000); hace énfasis en la introducción de la computadora dentro del aula, no como un elemento accesorio, sino como lo dice el mismo Martínez y otros (2006, p. 5) como un dispositivo importante que debe ir tomando un papel protagónico, en la reformulación del aprendizaje con nuevas herramientas tecnológicas, ya que la introducción de las Tic's en el aula en ocasiones no ha trascendido labores más significativas. Es decir, llegan a cumplir un papel marginal en el desarrollo didáctico y en el trabajo cotidiano de los alumnos. Sin embargo, se señala que entre los aspectos importantes que pueden facilitar su implementación, es la alfabetización tanto informacional como digital.

El estudio realizado por el mismo autor sobre la puesta en práctica, de este medio, por parte de los docentes, demuestra que su utilización se limita a la elaboración de trabajos de investigación, preparación de recursos didácticos, elaboración de guías metodológicas, confección de tareas y pruebas. Que entre los programas más usados se encuentra el procesador de texto de Microsoft Word, el Internet Explorer y buscadores como, Alta Vista o Yahoo. Así las actividades propuestas por ellos, según Martínez y otros (2006) se concentran en el aprovechamiento del procesador de textos, normalmente para las mismas tareas que antes se hacían por otro medio; en la búsqueda de información, ahora se agregan apoyos electrónicos a los tradicionales medios impresos. Por lo que se puede afirmar, que de acuerdo con resultados analizados, se hace necesario enseñar nuevas estrategias que permitan a los usuarios potenciar el uso de estos medios en el campo educativo y que lleven a un conocimiento más profundo de algunas metodologías que se pueden aplicar, a través de mediaciones didácticas 
que logren una aplicación más efectiva de estos medios en el campo curricular a través de la alfabetización tanto informacional como digital.

Todas estas apreciaciones anotadas, sobre el limitado manejo de la tecnología dentro del aula, evidencian la necesidad de una capacitación permanente y continua, tanto para docentes, como para estudiantes, sobre el apoyo de los medios tecnológicos en el desarrollo de sus actividades educativas en las que, se vuelve necesario, formar a los usuarios de estos medios, en el desarrollo de destrezas que favorezcan el proceso de enseñanza-aprendizaje, haciendo uso de esa herramienta. Así como, de contar con una infraestructura tecnológica adecuada y de un análisis profundo acerca del desarrollo de la alfabetización digital e informacional como instrumento para el desarrollo profesional.

Por otro lado, los estudiantes, son otros de los actores en el ámbito educativo que incursionan en el uso de las Tic's, pero al igual que los docentes, su implementación en el campo educativo es muy limitada. Normalmente la utilizan para realizar investigaciones asignadas por los docentes, y en su experiencia, según Martínez y otros (2006) encuentran dificultad para realizar las tareas educativas, pues muchas veces lo ven más como un aspecto lúdico o de entretenimiento que como un potencial para aprender. Sin embargo, los estudios realizados por Fazeli y otros (2000) demuestran la presencia de dos elementos que favorecen el aprendizaje: el nivel de receptividad y la motivación hacia el acto educativo mediante las Tic's.

Estudios, como el de López y otros (2006) se han enfocado en la exposición de algunos factores que intervienen en la aplicación de las tecnologías en el campo educativo, y que pueden propiciar una actitud favorable o de resistencia en la utilización de la misma. Entre los factores positivos mencionados por Martínez y otros (2006) se encuentran: la capacitación que han recibido, la experiencia que han tenido y la auto eficiencia, en el manejo de la herramienta. Todo esto lleva a la reflexión acerca de los cambios o modificaciones que han de darse para favorecer su uso en el proceso de enseñanza-aprendizaje con miras a fomentar la alfabetización tanto informacional como digital. Ya que, es importante no solo su introducción, sino que esta implementación vaya acompañada como afirma López y otros (2006, p. 5) de "cambios profundos en la estructura académica y administrativa, para lograr los fines deseados" siendo ésta, no solo una cuestión de buena voluntad por parte de los actores, sino que también de una gran disposición institucional que ponga en marcha todo un plan macro de acción para lograr cambios, en el campo administrativo.

Estudios españoles analizan la experiencia de docentes que han trabajado con la implementación de la tecnología; uno de ellos, es el de Matas y otros (2004) menciona que entre los aspectos más relevantes para el uso de los medios tecnológicos, es que éstos pueden ser facilitadores del proceso de enseñanza-aprendizaje en el aula, ya que las lecciones se vuelven más activas e innovadoras y mejoran la comunicación entre los alumnos y otros docentes de la institución, entre otros.

En consecuencia, analizando las diferentes formas en que se está aprovechando la tecnología en el campo educativo se plantea para el desarrollo de este artículo, la siguiente proposición:

"La elaboración de una propuesta curricular que permita alfabetizar tanto informacional como digitalmente al usuario de las Tic's; se deben considerar tres dimensiones: la tecnológica, la pedagógica y la administrativa".

Por lo tanto, se vuelve importante en el desarrollo de este análisis, el papel de la alfabetización, no solo digital sino sobre todo informacional, que destaque la relevancia de ser introducida en el medio educativo, como una herramienta que permita a los usuarios aprovechar el medio para la construcción de los conocimientos y 
el desarrollo de una mentalidad crítica que explote la red de forma racional.

De ahí que, el artículo contemple tres grandes apartados. En el primero se trabaja el concepto y características de la tecnología de la información y la comunicación y el papel de los docentes y los estudiantes como usuarios. El segundo presenta la perspectiva de la alfabetización tanto digital como informacional. Y el tercero plantea la forma en que se deben de desarrollar los elementos curriculares que deben de estar presentes en una propuesta curricular que haga uso de las Tic's (la mediación pedagógica, los objetivos, los contenidos, los recursos, la metodología, las estrategias didácticas y la evaluación) a partir de la alfabetización tanto informacional como digital.

\section{Las Tic's en el campo educativo.Concepto y características}

Para tratar la implementación de las Tic's dentro del campo educativo, es necesario primero plantearse qué se entiende por ellas. Estas, según Adell (1997, p. 3. Las nuevas tecnologías de la información y la comunicación, parr. 1) son:

(...) el conjunto de procesos y productos derivados de las nuevas herramientas (hardware y software), soportes de la información y canales de comunicación relacionados con el almacenamiento, procesamiento y transmisión digitalizados de la información.

En este sentido, se aborda el aspecto tecnológico, ya que se refiere directamente a aquellos elementos que hacen posible su uso, como al procesamiento que hacen de la información, los usuarios. Ahora, desde el punto de vista de los recursos, estos trascienden los que tradicionalmente se manejan en el campo educativo; según Bello (s.f., Escenarios educativos virtuales, párr. 8,9 y 10).

En lugar del lápiz, el cuaderno, los lápices de colores, y los libros de texto, el actual utillaje educativo del aula virtual está formado por la pantalla, el ratón, el teclado, la multimedia, la pizarra electrónica, el software y los videojuegos.

Los estudiantes deben aprender a manejar estos nuevos instrumentos y los creadores de materiales educativos deben plasmar el conocimiento y las destrezas en los nuevos soportes.

En las aulas virtuales se estudiará ante la computadora y con el CD (...)

A partir de este concepto, se hace necesario proyectar toda una tarea de alfabetización informacional y digital de los usuarios, que permita enseñar los principios que se refieren, al manejo de la tecnología, a la utilización y procesamiento de la información que hay en ellos. Cabe mencionar, que uno de los aspectos que favorecen su implementación en el proceso educativo es que se convierten en un apoyo didáctico del profesor al permitir la transmisión de conceptos, procedimientos, valores y actitudes desde otros medios que normalmente no se han utilizado, superando el uso superficial, marginado y limitado que se ha estado haciendo de ellos. Así, a los estudiantes, se les ofrece un acceso más fácil e interactivo de las fuentes de información y de conocimiento donde pueden desarrollar habilidades de expresión de forma más creativa y con nuevos medios y soportes (donde trabaje el aspecto informacional de los medios). También provee de escenarios de aprendizajes cooperativos, tanto presenciales como virtuales (foro, chats, correos) que permite al individuo familiarizarse con el uso de herramientas tecnológicas que podrá luego transferir a otros medios.

En fin, son muchos los aspectos positivos que provee este medio y las ventajas que ofrece ya que rompe con la concepción espacio y tiempo. Puede ser utilizada tanto, dentro como fuera del aula y en momentos diferentes lo cual permite el acceso a la educación a personas que no contaban con esa flexibilidad espacio-temporal. También ofrecen una serie de características que lo convierten en un gran potencial para enriquecer el trabajo educativo, de ahí la importancia de conocerlas para también aprovechar al máximo este medio. 
Características de las Tic's dentro

del campo de la alfabetización informacional y digital

La aplicación de las Tecnologías de la información la comunicación (Tic's) en el aula parte de tres grandes dimensiones según Bolaños (2007):

- la función tecnológica,

- la administrativa y

- la pedagógica, que se describen en la figura $\mathrm{N}^{\circ} 1$ :

Figura No. 1

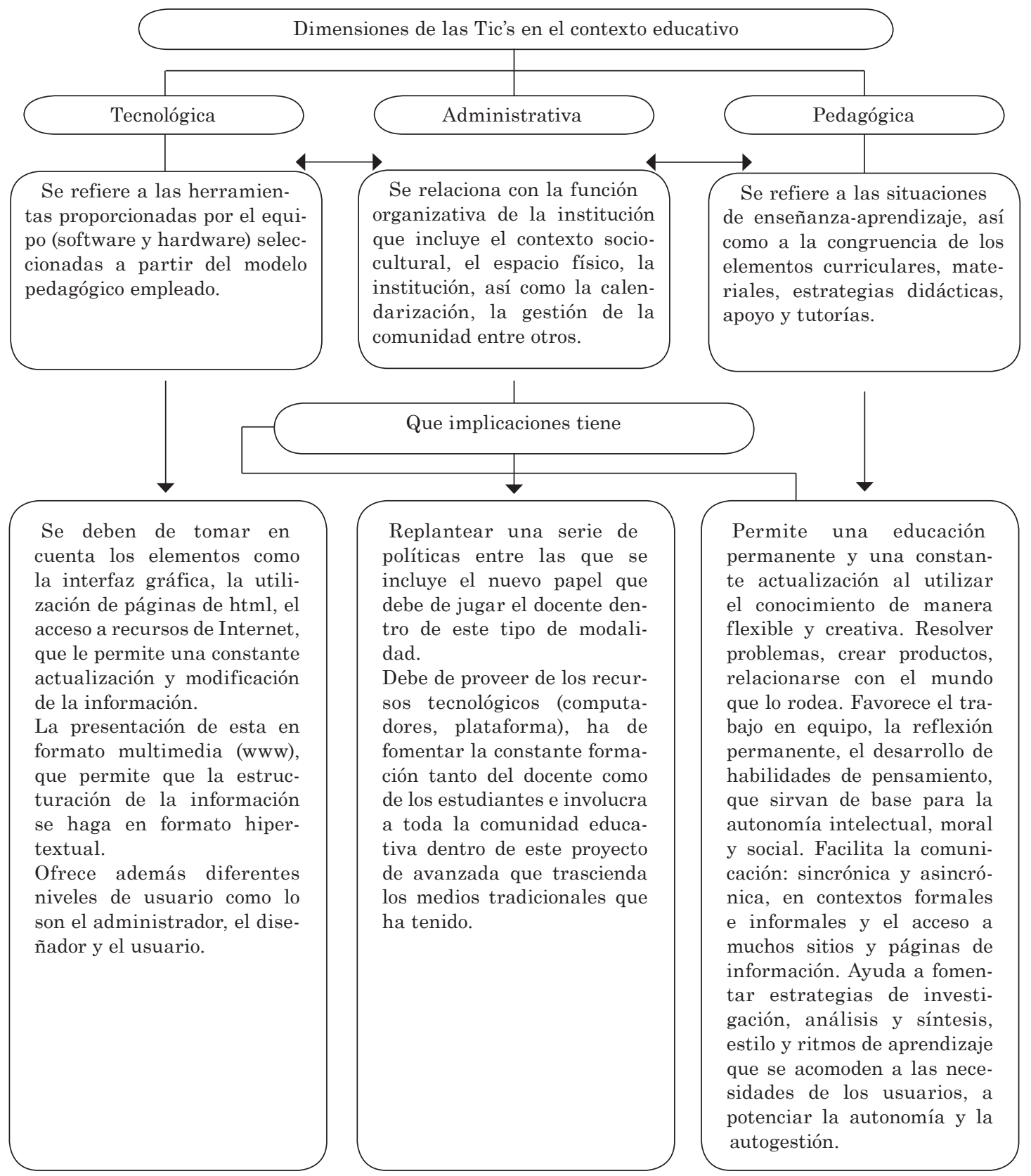


En la figura $\mathrm{N}^{\circ} 1$ se pueden ver las interconexiones que se establecen en las diferentes dimensiones, lo cual significa que una sin la otra dejaría incompleto el propósito ya que no podría cumplir con eficacia el modelo que se propone. No se trata tampoco de darle más importancia a una dimensión que a la otra sino que las tres son realmente necesarias para un desarrollo efectivo dentro del contexto educativo.

El papel de los usuarios, dentro de esta alfabetización tanto informacional como digital, juegan un papel relevante, es decir el docente y el estudiante, deben de desarrollar una serie de funciones en cada una de las áreas mencionadas, por lo cuál se planteará a continuación cuál es ese papel que deben de jugar dentro de este nuevo mundo educativo que aprovecha la tecnología.

\section{Usuarios de las Tic's}

Los dos principales agentes en la implementación de las Tic's dentro del campo educativo son: el docente y el estudiante.

Por lo tanto, es relevante estudiar cuáles son las principales funciones que cumplen estos dos agentes dentro del mundo de la alfabetización informacional y digital que se ha venido tratando.

\section{El docente}

\section{El rol del} docente varía de acuerdo con la concepción tradicional que se ha tenido de él porque no va a ser el actor principal dentro del proceso, sino que se convierte El docente que trabaja con las Tic's, debe de manejar un dominio básico de la tecnología, que le permita trascender su rol de simple informante y se convierte en un administrador del conocimiento. Desde esta perspectiva, rompe con el papel de simple usuario de herramientas computacionales sencillas, para convertirse en un agente que potencializa el medio para la adquisición del conocimiento.

en un ente que dirige y ayuda en el proceso al estudiante. Por eso es tan importante que se planteen procesos de formación y actualización para que pueda cumplir su papel, de forma efectiva y eficaz. Por lo tanto, como afirma Martínez y otros (2006, p. 19) "es necesario que se incorporen (...) seguimientos y apoyos que sirvan de andamiaje al docente...”. Esos apoyos tienen que ver principalmente con la información que pueda recibir sobre diferentes estrategias didácticas que puede implementar cuando utiliza las Tic's así como conocer cuáles serían las más adecuadas para el contenido que está abordando.

Ya dentro de las Tic's el docente se convierte en ese facilitador del proceso de alfabetización. Desde esta perspectiva debe

\section{a. Brindar atención individualizada. \\ b. Manejar grupos pequeños.}

De esta forma su labor involucra una serie de tareas como por ejemplo: dar seguimiento a las actividades de aprendizaje con el fin de retroalimentar los procesos, estar en contacto constante con los estudiantes que tiene a cargo y reformular el planeamiento cuando sea necesario y las horas que dedica a estas tareas, entre otras responsabilidades. En el proceso de enseñanza donde se utilicen las herramientas

Funciones del docente
Brindar atención
individualizada al
estudiante que requiere
de diversos apoyos en
la retroalimentación de
las tareas que le asigna
y del seguimiento del
proceso.
Manejar grupos
pequeños que le
permita conocer los
estilos y ritmos de
aprendizaje, así como los
conocimientos previos
que estos poseen sobre
el tema que se está
estudiando.
tecnológicas, ya que la persona que aprende haciendo uso de estos medios no desea dejar de contar con el acompañamiento humano en su aprendizaje. De ahí la importancia de preparar de forma adecuada a la persona que va a dirigir estos procesos para que interiorice su papel, por medio de capacitaciones donde se instruya sobre el nuevo rol que debe jugar en el manejo de este medio. 
Ahora bien, como se menciona, las dimensiones en que se va realizar esta tarea implica tres campos que deben ser tratados por el docente: el tecnológico, el pedagógico y el administrativo. En cada aspecto se señalan las competencias bási- cas, mencionadas por González y otros (s.f.) en la figura $\mathrm{N}^{\circ} 2$.

Esto lleva el desarrollo de ciertas competencias por parte del docente en este proceso anotados por el mismo autor, y que se dan en la Figura $\mathrm{N}^{\circ} 3$.

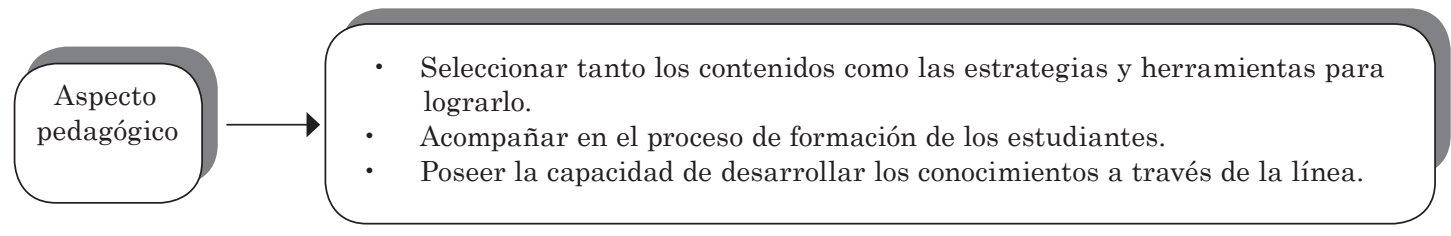

Figura $N^{0} 3$

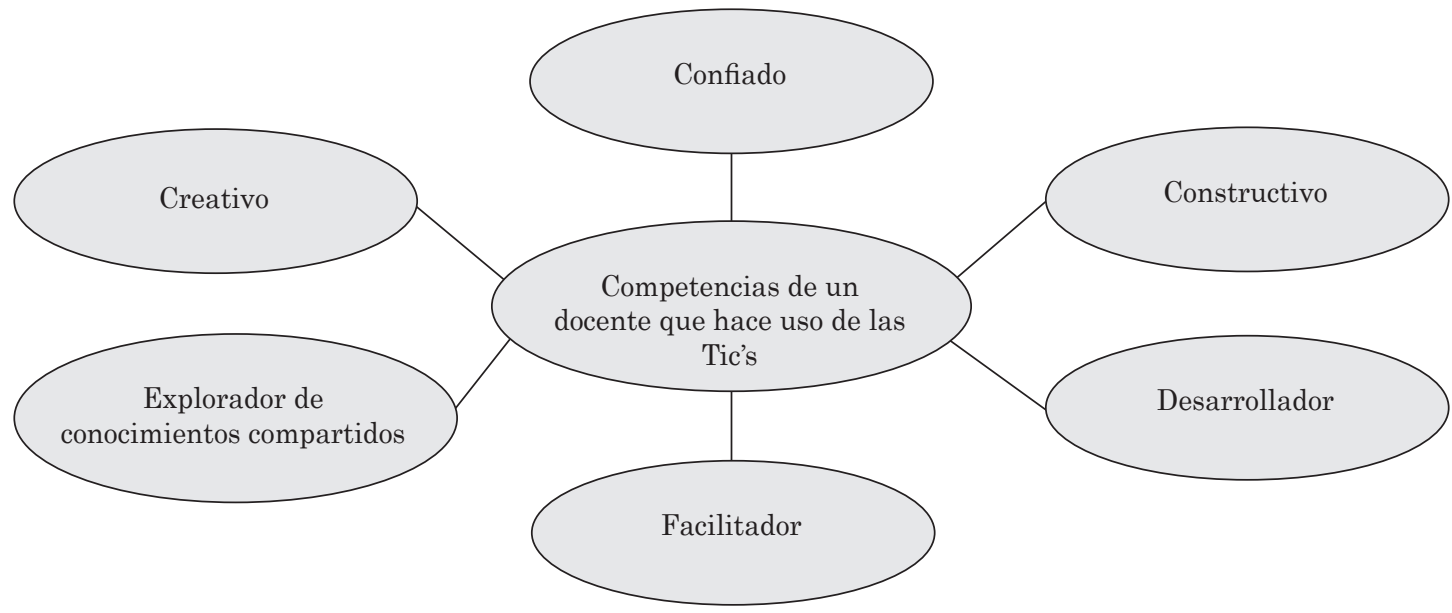

Todos estas competencias le permitirán, desarrollar sus estrategias de enseñanza haciendo uso de las Tic's de forma productiva y efectiva. Por lo tanto, el docente debe de ir más allá de un simple facilitador o contestador de preguntas, debe ser más bien un individuo capaz de generar en los estudiantes una fuerte motivación y guiar el proceso de forma efectiva así como proporcionar una visión del proceso de forma clara y precisa. También ayudar a los participantes a controlar sus frustraciones, ya sea en el manejo de la tecnología o en la comprensión de los contenidos.

En cuanto a los aspectos tecnológico y administrativo, según la figura $\mathrm{N}^{\circ} 4$, se tiene que 
Figura $N^{\circ} 4$

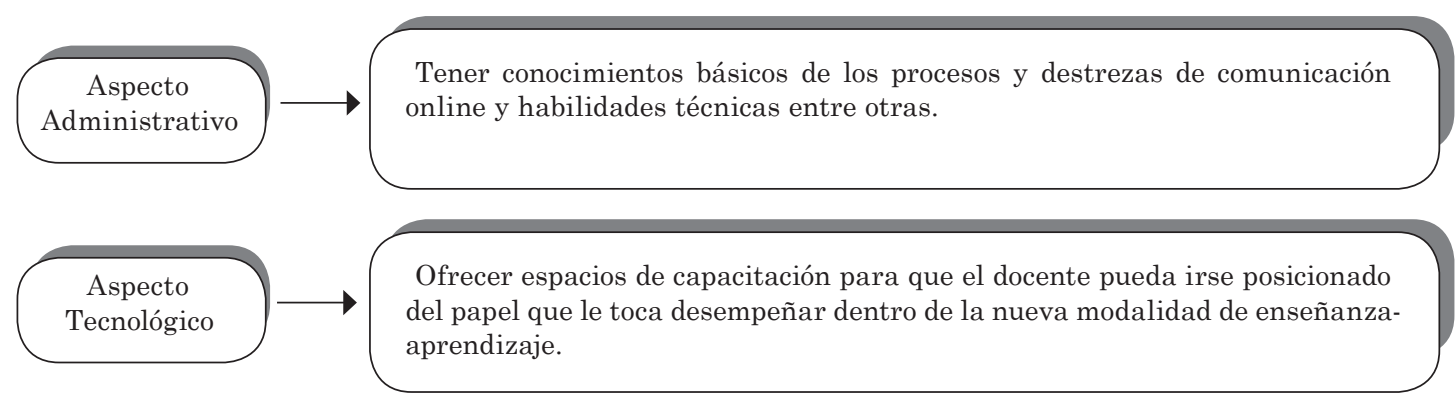

Con base en lo anterior se puede afirmar que un docente que se mueve en el ámbito de la alfabetización tanto informacional como digital debe poseer cualidades y habilidades tanto en el desarrollo de la tecnología como de comunicación para lograr una implementación efectiva en el proceso de enseñanza-aprendizaje.

$\mathrm{El}$ otro agente que interviene en este esquema es el estudiante. Con respecto a éste es importante hacer referencia a la forma en que se ve afectado en el uso de la tecnología en el campo educativo.

\section{El estudiante}

El papel del estudiante también se ve afectado cuando se enfrenta a la implementación de la tecnología y ésta trasciende el uso superficial y limitado para hacerlo de una manera más efectiva y concreta. Cuando lo asume, se convierte en un participante activo dentro del ambiente educativo que incorpora el recurso tecnológico en el aprendizaje, por lo tanto, la alfabetización tanto informacional como digital, se convierte para él en un medio sumamente importante, no solo en su dimensión del manejo de la tecnología sino también por el desarrollo de destrezas en la comunicación.

En este sentido Cabero (1994, p. 6) menciona que desde la dimensión pedagógica, cuando los estudiantes hacen uso de los medios tecnológicos se está potencializando:

(...) el desarrollo de destrezas como la planificación, el trabajo colaborativo en equipo, el aumento de la motivación hacia los contenidos, la comprensión del funcionamiento técnico de los medios, el desarrollo de habilidades de comunicación escrita, el progreso en el desarrollo de la comunicación oral, la adquisición de destrezas sociales, los cambios en las relaciones profesor-alumno.

De lo anterior se puede inferir que el manejo de las herramientas tecnológicas permite al estudiante aprender a procesar la información que recibe y a desarrollar una serie de destrezas sociales y de comunicación.

Por esta razón, Marquès (2000) menciona algunas de las funciones que tiene desde el punto de vista pedagógico el aprendizaje apoyado en las Tic's dentro de la alfabetización informacional las cuales se anotan en la figura $\mathrm{N}^{\circ} 5$ :




Así el proceso de aprendizaje busca ir más allá de la sola trasmisión de conocimientos porque se orienta más bien a la búsqueda de estrategias que buscan procesar el significado de los contenidos mediante un análisis de los mismos. Entre las implicaciones de esta dimensión se encuentra el hecho de que el estudiante debe aprender a manejar dos tipos distintos de representaciones, acotadas por Onrubia (2005, p. 4).

(...) representaciones sobre el significado de contenido a aprender. Y por otro representaciones sobre el sentido que tiene para él aprender ese contenido, sobre los motivos para hacerlo, las necesidades que ese aprendizaje cubre y las consecuencias que supone para la percepción de uno mismo (...)

Por lo tanto, como se ve, un uso adecuado de este medio plantea ir más allá de la sola transmisión de conocimiento ya que más bien pretende que el estudiante también analice sus motivaciones para aprender, lo cual, hará más significativo el aprendizaje.

La alfabetización digital e informacional según Bolaños (2007), debe permitir al estudiante desarrollar, una serie de destrezas tecnológicas, tales como las anotadas en la figura $\mathrm{N}^{\circ} 6$.

Como se muestra en la Figura $\mathrm{N}^{\circ}$ 6 el estudiante será capaz de desarrollar una serie de destrezas que le permitirán un desempeño óptimo cuando se enfrenta a la tecnología en el campo educativo. Para ello la utilizará como una herramienta o un medio no como un fin en sí mismo. Se debe destacar que el manejo de las Tic's en el campo educativo debe de partir de una decisión institucional que disponga de la infraestructura tecnológica al servicio del estudiante, así como de inversión en

Figura $\mathrm{N}^{\circ} 6$

El envió de información o inquietudes que le surjan tanto al profesor o el poder compartirla con sus compañeros.

Navegar en red en la búsqueda, selección y recuperación de información que le sea útil en el cumplimento de los objetivos propuestos en el curso.

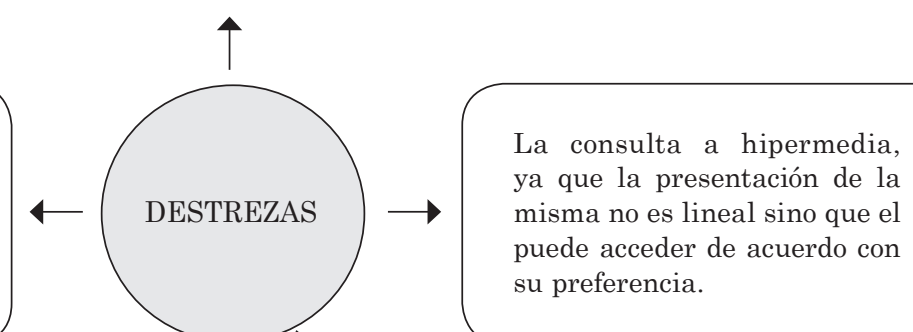

La comunicación constante y fluida con el profesor a través de la tecnología, que permita la retroalimentación en el proceso de aprendizaje para el logro de los objetivos propuestos.
Participación activa en las actividades de tele presencia que se programen con el fin de posibilitar relaciones con otras personas que también acceden a esos medios. 
recursos humanos donde ofrezca personal competentemente preparados en el aspecto técnico y pedagógico para desarrollar exitosamente esta nueva opción.

En consecuencia, debe trabajarse desde un nuevo enfoque ya que, como usuarios desde la perspectiva educativa, cobran una dimensión protagónica en el proceso de enseñanza-aprendizaje.

Este nuevo rol trasciende el manejo superficial, marginal y limitado que se está haciendo de la tecnología para trabajar más bien niveles más complejos que les permitan estar al alcance de las exigencias de la sociedad actual.

Aunque se ha venido desarrollando el tema de la alfabetización tanto digital como informacional es necesario conceptualizar ambos términos y determinar sus implicaciones.

\section{Alfabetización digital e informacional}

La alfabetización digital e informacional se ha vuelto cada vez más imperativa en una sociedad que cada vez más está conectada a la tecnología de la información y la comunicación en todos los ámbitos y que por lo tanto tiene no solo el reto de preparar al usuario en el uso digital de ésta sino de permitirle desarrollar también destrezas que le faciliten el manejo de la información que ésta contiene.

Según Bawden, en Silvera, (2005, p. 2) el concepto de alfabetización

\footnotetext{
"va mucho más allá de saber leer y escribir; que constituiría una [manera] básica [de que las personas aprendan aspectos del lenguaje o del manejo de operaciones matemáticas básica]. En las últimas décadas, este concepto se ha complementado con las llamadas "alfabetizaciones en destrezas", conceptos desarrollados para hacer referencia a una información de creciente complejidad y a las tecnologías en auge.
}

Esto permite afirmar que cuando se menciona la alfabetización tanto digital como informacional, se hace alusión a una serie de destrezas que son necesarias que el individuo desarrolle en el uso de la tecnología de la información y la comunicación.

La alfabetización digital, se refiere, según Bawden, (2002, pp. 370-371) a la

(...) introducción en aquellas destrezas que se requieren para poner en marcha un conjunto de paquetes de aplicaciones informáticas - procesamiento de textos, bases de datos, hojas de cálculo, etc.- junto con algunas destrezas generales propias de las Tic's, como el grabar en diskettes, o generar un documento impreso. El texto presenta estas destrezas en un formato estructurado, con revisión inicial, ejercicios de consolidación, listas de revisión de órdenes, tests de autoevaluación y pruebas...

Desde esta perspectiva la alfabetización digital implica, por parte del usuario, la puesta en práctica de una serie de habilidades para manejar los programas de computación que le permitan tener acceso a la información.

Se refiere por tanto a la adquisición de conocimientos prácticos, destrezas y actitudes que debe ser empleada por el docente y el estudiante frente a los ordenadores. También se puede decir, que se refiere, a la utilización de instrumentos como el hardware, el software y los programas multimedia y recursos informacionales que están en la red que les permita la publicación y la difusión de productos. Asimismo implica, actualización en relación a la tecnología de punta que se ofrece en el mercado, sobre todo a los paquetes computacionales que constantemente se renuevan.

Según el documento de Normas sobre Alfabetización en Información, el concepto de la alfabetización informacional se refiere a la "capacidad de comprender y un conjunto de habilidades que capacitan a los individuos para "reconocer cuándo se necesita información y poseer la capacidad de localizar, evaluar y utilizar eficazmente la información requerida" (Council of Australin University Librarians, 2002, p. 68).

Desde este punto de vista, la alfabetización informacional requiere que el 
usuario adquiera una serie de destrezas que le permitan tener un acceso más eficaz y efectivo a la información. Bruce (2003) hace referencia a algunas de las implicaciones que conlleva este tipo de alfabetización, que se cita en la Figura $\mathrm{N}^{\circ} 7$.

Figura $\mathrm{N}^{0} 7$

Como ejecución de un proceso: Afrontar situaciones nuevas que le permitan establecer procesos para encontrar y utilizar la información necesaria.

Para hallar información: Conocer cuáles son las fuentes y su estructura, cómo usarlas con independencia o ayudado.

Ofrecer el control de la información: Recuperar información que ha almacenado, en el momento en el que la vaya a necesitar.

Ayudar a la recuperación y comunicación de la información ya que en la actualidad el volumen que esta ofrece, es realmente enorme por lo cual es necesario crear estrategias que faciliten el acceso a la información con mayor agilidad y efectividad.

Como extensión del conocimiento: Producir nuevos conocimientos que pueda ser divulgado y que permita ir de una sociedad de la información a una sociedad del conocimiento.

Como construcción del conocimiento: Ejercitarse en la evaluación y el análisis de los documentos que utiliza y que trascienda el uso solo de consumir del conocimiento, se debe de convertir también en un productor del mismo.

En la utilización de la información en beneficio de los demás: Poner en práctica una serie de operaciones como la emisión de juicios, la toma de decisiones y la investigación dirigida a buscar el beneficio de los demás por encima del propio.

Si vemos en esta figura $\mathrm{N}^{\circ} 7$ podemos ver que se desarrolla un proceso que inicia desde habilidades para encontrar y localizar la información hasta ser capaz de construir conocimiento y una vez que se ha elaborado ponerlo a la disposición de la comunidad en general. Por lo tanto cada una de las etapas son importantes e indispensables, porque no se puede producir conocimientos si no se han desarrollado las destrezas necesarias para encontrar la información ya que debido a su amplitud y complejidad no podría manejarse fácilmente, ahí la importancia de conocer y desarrollar 
las estrategias que permitan llegar a la fuente de información que se requiere para construir el conocimiento.

Por otro lado Fainholc (2004), menciona que cuando se está utilizando en el ámbito educativo las Tic's es necesario conocer las diferentes dimensiones sobre las cuales se está trabajando la mediación. Entendida ésta como el intercambio que se produce entre los usuarios de las Tic's. En este sentido, se está evidenciando que hay una serie de repercusiones que se producen cuando se está ante el uso del medio tecnológico en el ámbito pedagógico. Por lo tanto, esta relación que se establece en la utiliza- ción de estos medios se evidencian cuatro grandes campos: cultural, comunicacional, semiológica y tecnológica. Cada una de estas dimensiones interactúa de manera que no se puede desligar una de la otra como se observa en la figura $\mathrm{N}^{\circ} 8$, donde se caracteriza cada una de ellas de manera que se pueda tener una idea de cuales son esas interconexiones que se establecen entre los usuarios de las Tic's y donde se evidencia una interconexión entre las diferentes dimensiones, ya que no se dan de forma aislada, sino que se relacionan entre sí para conformar entre sí la mediación en su aspecto más amplio.

Figura $N^{0} 8$

Mediaciones con las Tic's

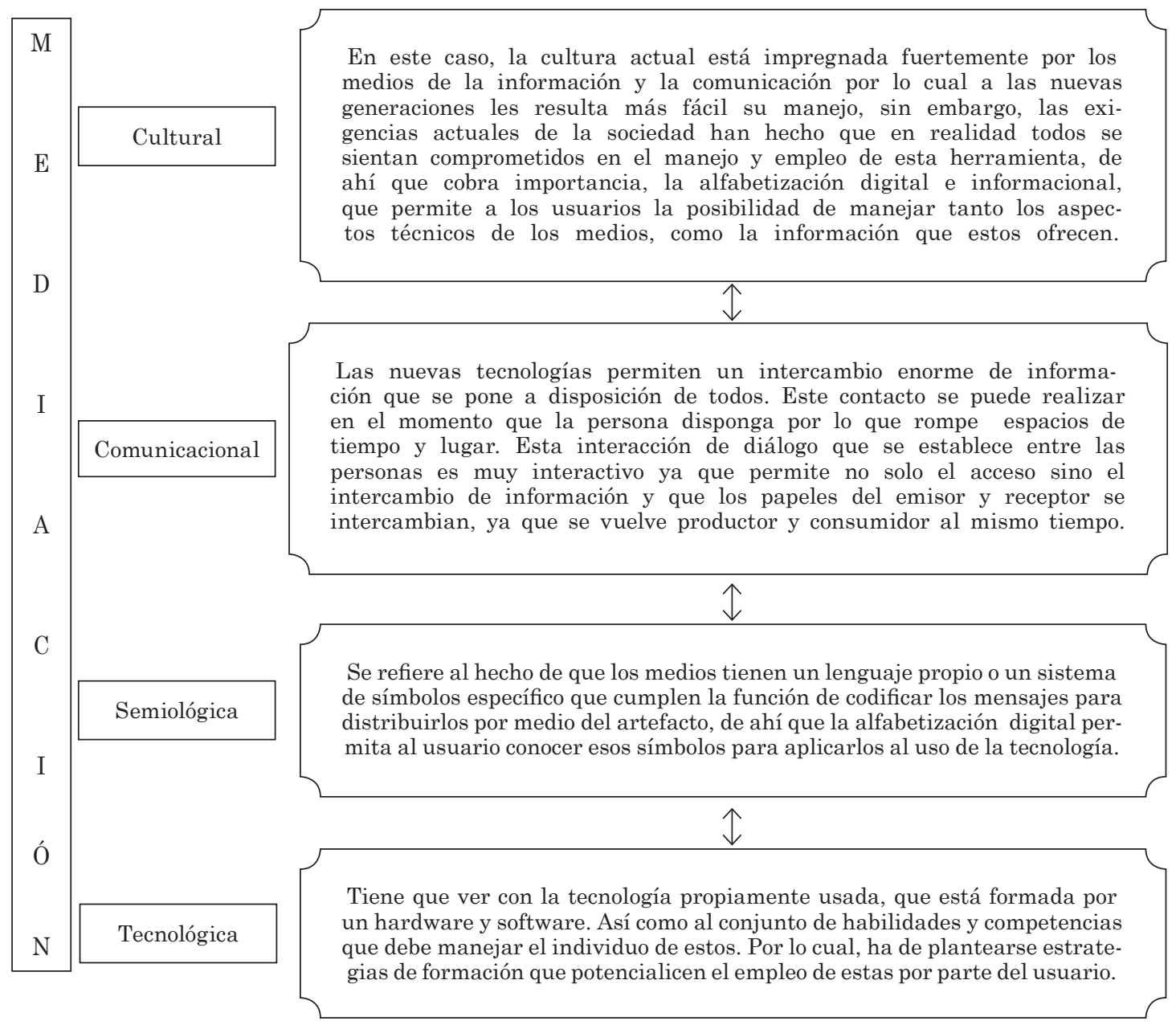


Por esta razón el usuario ha de ser formado en el manejo de herramientas que le permitan desempeñarse de forma eficiente en los diferentes ámbitos. Es en ese sentido donde cobra gran importancia que el campo educativo sea uno de los primeros que impulse esta formación ya que se vuelve imperativo la necesidad de dar a los docentes y estudiantes las herramientas necesarias para que se incorporen a la sociedad y hagan frente a los retos de las nuevas demandas en el campo tecnológico, de la digitación y la información. Hasta este momento se ha tratado el aspecto tecnológico y administrativo en los diferentes aspectos que compete al usuario de las Tic's. Ahora el análisis que se realiza se centrará en el pedagógico con el fin de ofrecer una ayuda en el aspecto de la gestión curricular que permita a estos potenciar su empleo en el campo educativo.

\section{Potencial pedagógico de las Tic's y la planificación curricular}

$\mathrm{Al}$ inicio del trabajo se evidenció cómo el manejo de las Tic's en el campo educativo se realiza de forma muy superficial marginal y limitada y que para superar esta visión se vuelve necesario llevar a cabo una labor de alfabetización digital e informacional que permita a los usuarios aprovechar todo el potencial que éstas ofrecen en el campo pedagógico.

Por ejemplo, en el caso de los recursos, las imágenes favorecen el aprendizaje ya que muestran lo que se explica, no solo con palabras, sino que permite percibir lo que otros medios como los escritos no logran hacer con igual realismo cuando se aprovecha el recurso visual. También, en el manejo de los elementos tecnológicos se pueden ofrecer diseños atractivos que propician experiencias que enriquecen el aspecto conceptual y permiten organizar los conocimientos de formas más adecuadas. Otro de los factores que menciona, como favorecedores Cabero (2002): el aprendizaje, es la fuerte interactividad que mantiene la atención constante del educando sobre la actividad que se realiza. En este sentido Fainholc, menciona el aporte de Gadamer, H., (2004, p. 3) sobre

[los] elementos [que intervienen en ese] "diálogo" porque el intérprete y el texto son dos interlocutores que a través de la articulación dialéctica de preguntas y respuestas relacionadas mutuamente, pretenden alcanzar el entendimiento para comunicar algo.

Se vuelve relevante el aprovechamiento de esa interactividad, para la construcción del conocimiento que debe ser propiciado en el campo educativo. Para que esta dimensión pedagógica se cumpla de forma efectiva es importante ahondar en aspectos de planificación curricular tales como la buena elección de los objetivos y los contenidos que se va a trabajar en el curso, el material que se va a utilizar en los que se toma en cuenta criterios como la calidad y actualización; que las actividades de aprendizaje sean las pertinentes para el desarrollo del contenido, así como una buena congruencia entre los objetivos del curso y los contenidos desarrollados. Se deben plantear claramente los criterios de evaluación y la retroalimentación continua, así como formular realmente los tiempos destinados para las actividades. Todo esto será posible en la medida en que los usuarios sean capaces de manejar la tecnología desde los dos puntos de vista que se han tratado: el digital y el informacional. De ahí, que el aporte de este artículo es demostrar que para elaborar una propuesta curricular que permita alfabetizar tanto informacional como digitalmente a los usuarios educativos de las Tic's es necesario considerar las tres dimensiones estudiadas: la tecnológica, la administrativa y la pedagógica. Desde el punto de vista de esta última es necesario tomar en cuenta los elementos curriculares de programación: los objetivos, los contenidos, los recursos, la metodología, la didáctica y la evaluación los cuales deben 
estar orientados a favorecer el aprendizaje efectivo.

Es importante hacer hincapié que en la formulación de un planeamiento curricular que busque aprovechar las Tic's debe tomarse en cuenta que los objetos para el aprendizaje no serán diferentes a los que tradicionalmente se han utilizado pero que necesita que el estudiante y el docente sean capaces de usar la tecnología sin ninguna limitación, de ahí que se tome en consideración la formación que propicie las posibilidades de hacer uso de éstas, de forma clara y precisa. Surge por lo tanto la necesidad de hacer referencia a los elementos curriculares relacionados con la programación como son los objetivos, contenidos, estrategias metodológicas y evaluación de los aprendizajes.

\section{Los objetivos}

La planificación debe partir de un propósito y fin específico. Los objetivos se convierten en el camino que se va a tra-

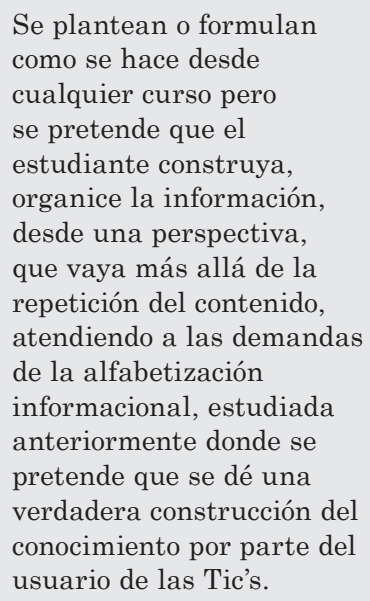

la mera repetición de éstos.

\section{Los contenidos}

Son aquellas unidades temáticas diseñadas de forma coherente y precisa cer cuáles son los logros por alcanzar. Se convierten en acciones que se esperan alcanzar con el aprendizaje de algún tema específico. Es importante tomar en consideración que se busca una construcción del conocimiento no para lograr el aprendizaje de un aspecto específico planteados en la construcción de cualquier diseño de curso.

Estos deben cumplir con ciertos requerimientos, como por ejemplo: ser claros, concretos, precisos. Ser actuales y contar al máximo con un grado de profundidad y amplitud en cuanto a
Se plantean tomando en cuenta el juicio del experto, las necesidades de lo que se espera aprender y la pertinencia del saber que se quiere alcanzar. De ahí que se haga una selección seria y meditada de lo que se espera aprender en relación con el tema seleccionado. los niveles de calidad y cantidad.

Si se presenta de forma coherente y logrando un desarrollo en el nivel de los contenidos que pase de aspectos básicos y generales a otros más profundos y complejos que permitan al estudiante, de forma ordenada y coherente lograr un aprendizaje significativo.

Josemaría (2001) propone algunos elementos a considerar para estructurar el contenido de un curso como por ejemplo:

zar para cono-

- Elegir una temática: bajo una serie de criterios didácticos, sin caer en aspectos solo descriptivos.

- Analizar los conceptos: no solo enunciarlos o hacer descripciones verbalistas.

- Detectar los conceptos y comenzar a construir en relación con conceptos de otros continentes epistémicos.

- Proyectar de forma global que superen la visión por áreas, donde se logre establecer elementos conectivos reales y ciertos.

- Seleccionar y analizar los contenidos en que se deben dar las conexiones deseadas.

- Elegir los recursos que se van a utilizar para trabajar ese contenido desde un fin didáctico y no simplemente por rellenar espacio.

- Seleccionar los textos que va a incluir, tomando en cuenta que sean pertinentes al contenido. 
También, es oportuna la reflexión minuciosa del contenido que se va a utilizar a través de la tecnología y aprovechar las destrezas que incluye la alfabetización informacional para el tratamiento y asimilación del tema que se tratarán.

\section{Los recursos}

Se entienden como todos aquellos materiales mediatizados como el video, el multimedio, la videoconferencia, los documentos en línea entre otros; previamente

Sin pretender realizar una
lista exhaustiva de los
recursos que la tecnología
ofrece en el campo de la
alfabetización digital que
pueden ser aprovechados
dentro del campo educativo
se encuentran los
siguientes:
- Material hipermedia:
$\quad$ con ingreso a una
temática, explicativas
$\quad$ que incorpore lo
sensorio-sensual.
Material hipertexto
útil para la
construcción de
concepto, a través de
las digitaciones donde
se puede trabajar con
el movimiento.
para potenciar de forma efectiva el aprendizaje, lo cual debe quedar claramente plasmado en el diseño del planeamiento.

Algunos ejemplos de recursos que se pueden utilizar son:

- Mensajería instantánea.

- Lista de distribución de correo y lista de discusión.

- Webquest. Navegadores y buscadores.

- Portafolio electrónico.

- Mediatecas y guías sociales.

- Catálogo de recursos.

- CD-ROM, DVD-ROM.

- Programa de software.

- Televisión interactive.
- World Wide Web, Blogs.

- Wikis, Portal educativo.

- Teléfono WAP, Correo electrónico.

- Grupo de noticias, Internet Relay Chat.

- Entornos abiertos (www).

- Compiladores por módulos, noticias.

- Enlaces a la web: ofrecer también herramientas de localización y acceso a información.

- Pizarras interactivas, ejercicios y pruebas de evaluación y archivos de video o sonido.

- Editores de páginas web, o de herramientas de autor, authoware professsional, macromedia, directorr, Toolbook.

- Herramientas para la creación de ejercicios y exámenes.

En la exposición de los materiales se debe considerar aspectos tanto de contenidos como aspectos gráficos y editoriales ya que es necesario que este tenga su impacto en la calidad y en la presentación. En este sentido, es necesario el trabajo en equipo con los encargados de producción que puedan ofrecer una guía para la implementación de este material porque aunque se tengan conocimientos básicos sobre el uso de la tecnología dados por la alfabetización digital, es necesario contar con el apoyo de los técnicos para la elaboración de materiales más complejos.

Algunos detalles que se deben visualizar tiene que ver con la presentación del sitio, el orden de la información, el tipo de formato de letra, los elementos de atractivo visual y colores entre otros.

\section{Estrategias metodológicas del aprendizaje}

Las estrategias metodológicas se refieren a las acciones que debe realizar el estudiante y que son planteadas por el docente para lograr que éste llegue al logro o cumplimiento de los objetivos. Estas son entonces, diversas opciones de aprendizaje 
que planea el docente para que el estudiante las ejecute y logre el fin esperado.

Algunas estrategias que el docente puede implementar mediante el uso de la herramienta tecnológica en la planificación curricular, propuestas por Cabrera (s.f.) son las siguientes:
El docente plantea actividades que realmente permitan potenciar el aprendizaje en el diseño de un curso que implemente el uso de las Tic's, por lo que debe partir de una posición reflexiva de cuáles son las más adecuadas. Algunas de las preguntas que se pueden formular son: qué estrategia es la más conveniente para lograr alcanzar el objetivo concreto, si ésta va a permitir abordar el contenido de forma clara y precisa y como estimar el tiempo que dispondrá el estudiante para lograr realizar la actividad.

- Procesos de lecturas y análisis de texto

- Uso de correo electrónico para consultas o compartir conocimientos.

- Intervención en televisión interactiva.

- Participación en actividades que le permitan leer, navegar e interactuar con el contenido propuesto.

- Aportación en foros de discusión con el fin de promover el ambiente colaborativo entre los estudiantes, estableciendo claramente las reglas y procedimientos mínimos para su ejecución.

- Ofrecer espacios donde los estudiantes lancen preguntas sobre temas concretos con el fin de favorecer la interacción en línea.

- Actividades que ofrezcan un aprendizaje significativo donde se les enfrente a situaciones desestructuradas, para tener la experiencia de organizarlas, participación de enlaces en subgrupos.

- Elaboración de tareas, envío de correo, evacuar preguntas y dudas, asignaciones de investigaciones o tareas, envío de documentos, entre otros.

\section{Estrategias didácticas}

$\begin{array}{ll}\text { Las estra- } & \text { Al conocer las herramientas } \\ \text { tegias didác- } & \begin{array}{l}\text { propuestas por la } \\ \text { alfabetización informacional } \\ \text { ticas se refie- } \\ \text { ren a aquellas } \\ \text { ser capanante podrá }\end{array} \\ \text { actividades que } & \text { síntesis y análisis con } \\ \text { han de ser eje- } & \text { los materiales que le } \\ \text { cutadas por el } & \text { proporciona el profesor o } \\ \text { estudiante en el } & \text { que consigue en la red, } \\ \text { proceso de ense- } & \text { participar en la formulación } \\ \text { nananta de preguntas } \\ \text { zaje, para que } & \text { en línea sobre aspectos } \\ \text { participe en en } & \text { particulares sobre el } \\ \text { forma activa en } & \text { contenido estudiado, estar } \\ \text { la construcción } & \text { constantemente informado } \\ \text { de las actividades del curso. }\end{array}$
aprendizaje.

Algunas estrategias didácticas que se pueden implementar son las siguientes:

- Consultar el directorio de usuarios.

- Realizar enlaces a la web.

- Participar en tutorías en línea.

- Participar en los grupos de discusión.

- Realizar vínculos propuestos en el medio.

- $\quad$ Participar en videoconferencias.

- Asistir a trabajos en grupos virtuales (colaboración).

- Construir un proyecto final.

- Elaboración de trabajos en grupos.

- Participar en actividades como: correo electrónico, chat, pizarra compartida, videoconferencias, transparencia de ficheros, entre otras.

\section{Evaluación sumativa y formativa}

Entendido este proceso como el seguimiento que se brinda para saber si se están alcanzando los objetivos planteados dentro de un modelo formativo y de acompañamiento.

Esta función de la evaluación según Cabrera (s.f., p. 11) "se dirige no solo a los 
productos del nivel de desarrollo real, sino sobre todo a determinar el nivel de desarrollo potencial (...)" planteada según la autora como una evaluación dinámica.

Sin embargo, es necesario reflexionar sobre los criterios y procedimientos de evaluación pues es una etapa muy importante dentro del proceso de formación que incluye dos formas: la formativa y la sumativa.

La evaluación formativa buscará favorecer la reflexión y la evaluación de los alumnos de su propio aprendizaje, donde lo que se pretende es orientarlos sobre su propio progreso a través de los ejercicios que se le ofrezcan para la autoevaluación. En este sentido se considera la evaluación diagnóstica como parte del proceso inicial y se parte de la premisa de la retroalimentación constante del estudiante en su proceso formativo. En la figura $N^{\circ} 9$ se detalla cada una de las acciones esperada en los diferentes momentos de la evaluación formativa.

Figura $N^{\circ} 9$

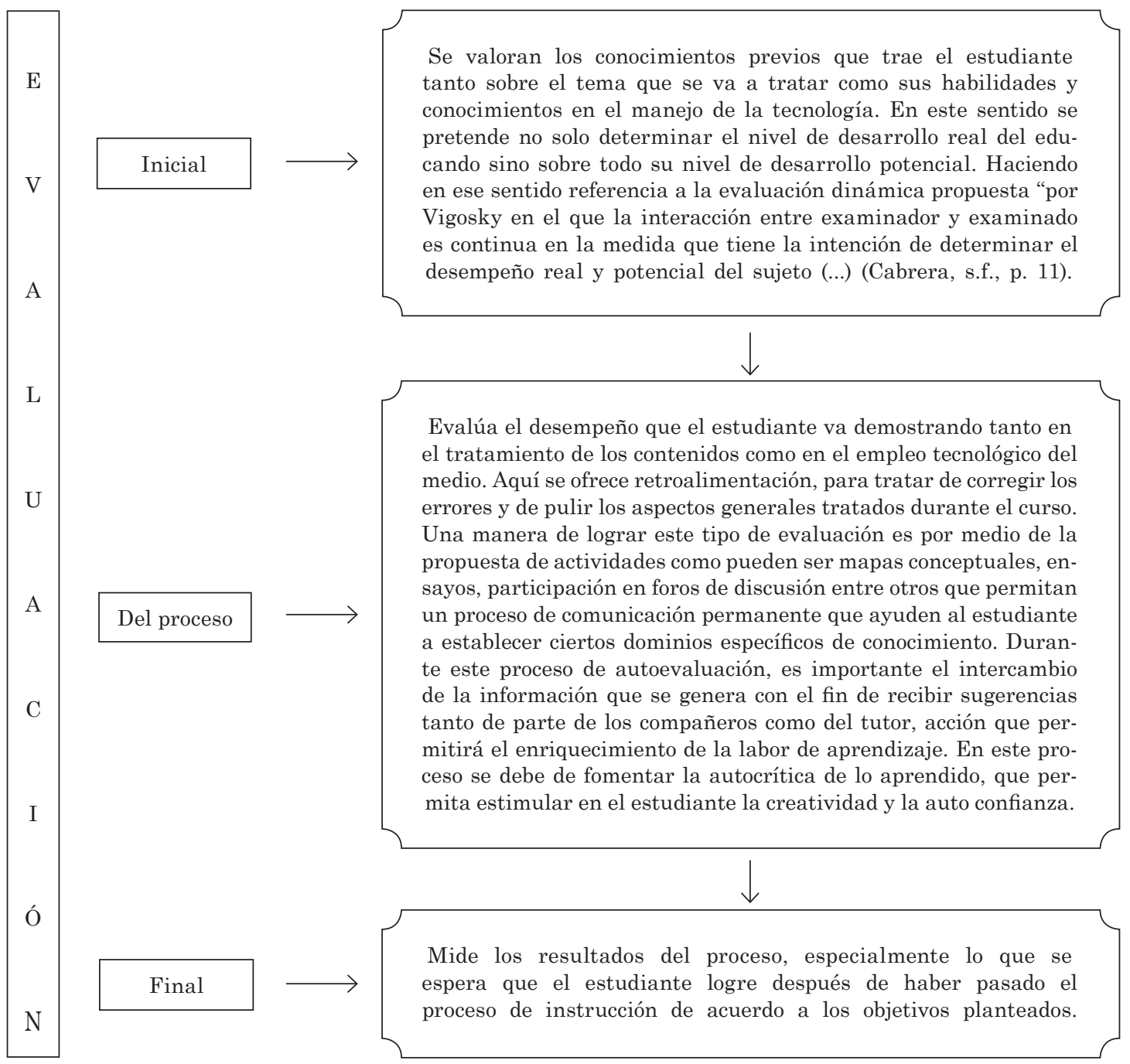


La evaluación sumativa responde a la asignación de puntos que se realiza a las tareas fijadas al estudiante. Esta ha de responder a algunas interrogantes que tienen que ver con aspectos que se van a evaluar (al cómo, cuándo y por qué). El docente es el responsable de organizar las actividades de evaluación al inicio de un curso con este tipo de modalidad y esta información se debe dar al estudiante para que conozca de antemano los aspectos que se le evaluarán. En la figura $\mathrm{N}^{\circ} 10$ se anotan algunos aspectos que el docente debe informar a los estudiantes, en cuanto a la evaluación.

\section{Figura $\mathrm{N}^{\circ} 10$}

Aspectos que se le deben comunicar al estudiante relacionado con la evaluación

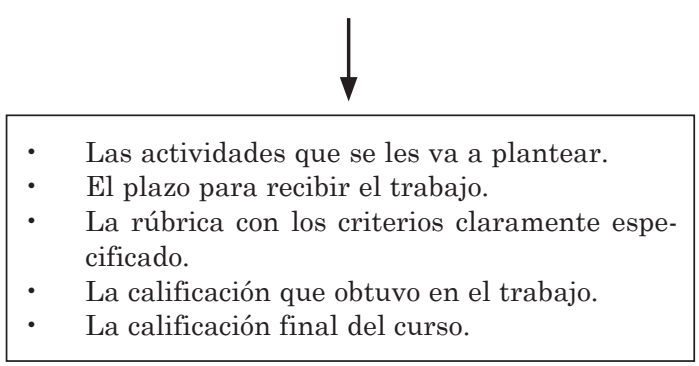

Las diferentes estrategias de evaluación deben de estar claras como por ejemplo reportes de trabajo continuo, foros de discusión y tareas definidas entre otras. También se ha de contemplar la asignación de un trabajo práctico final donde se pueda aplicar lo expuesto en el curso.

\section{Conclusión}

El trabajo deja claro cómo la implementación de las Tic's dentro del campo educativo es un factor de gran ayuda en el proceso de enseñanza-aprendizaje ya que puede proponer estrategias que propicien la construcción más que solo la trasmisión de los conocimientos. Otro de los aspectos que favorecen el aprendizaje utilizando las Tic's es que los estudiantes muestran una mayor receptividad y motivación para aprender. En cuanto a los docentes tenemos que en el uso de las Tic's se ve favorecido cuando cuenta con experiencia previa sobre el uso de ellas, la autoformación o las capacitaciones que han recibido. Esto ha permitido que muchos lo implementen como apoyo didáctico provocando lecciones más atractivas e innovadoras, que mejoran las relaciones, que fomentan ambientes más cooperativos y que favorecen el aprendizaje en todo momento pues rompen la concepción de espacio y tiempo. También permite un acceso más fácil e interactivo de la información y un desarrollo de otras habilidades como el uso de la tecnología y de la expresión.

Por todas estas razones surge la necesidad de realizar un planteamiento curricular que contemple los diferentes elementos internos de forma integral.

De acuerdo con los aspectos presentados la implementación de las Tic's es una realidad dentro del ámbito educativo pero surge la imperiosa necesidad de crear un diseño que permita orientar a los principales usuarios en un manejo efectivo de los mismos y donde estén presentes los tres aspectos estudiados: el tecnológico, el pedagógico y el administrativo.

Dentro del aspecto tecnológico se plantea la necesidad de la alfabetización digital donde el usuario tenga la capacidad de utilizar la herramienta de forma clara y eficiente.

Por otro lado también se plantea el desarrollo de la alfabetización informacional donde uno de los campos más importantes es el educativo, el docente y el estudiante van a trabajar desde una perspectiva de mayor participación y construcción del propio conocimiento.

$\mathrm{Y}$ por otro lado, aunque el aspecto administrativo fue poco abordado, sí se plantea la necesidad de que la institución provea los medios necesarios para que los 
usuarios tengan el acceso tecnológico necesario y prepare al personal para el uso de estrategias curriculares que la hagan efectiva en el campo educativo.

\section{Referencias bibliográficas}

Adell, J. (1997, Noviembre). Tendencias en educación en la sociedad de las tecnologías de la información. EDUTEC Revista Electrónica de Tecnología Educativa, 7. Extraído el 26 de abril 2008 de http://www.nti.uji.es/docs/ nti/Jordi_Adell_EDUTEC.html

Ariza, G., E. y Rouquette, A. J. (s.f.). Uso de un sistema tutorial inteligente en el ámbito educativo. Extraído el 2 de febrero del 2007 desde www.somece.org.mx/simposio2004/ memorias/grupos/archivos/080.doc

Bawden, D. (2002). Revisión de conceptos de alfabetización informacional y alfabetización digital. Anales de Documentación, 5, 361-408. Extraído el 20 de julio de 2007 de http://www. um.es/fccd/anales/ad05/ad0521.pdf

Bello, R. E. (s.f.). Educación virtual: aulas sin paredes. Extraído el 30 de junio 2007 desde http://www.educar.org/ articulos/educacionvirtual.asp

Bolaños, I. (2007). Herramientas telemáticas para la Enseñanza. En Plan de capacitación: Técnicas de colaboración en red para docentes. San José: Convenio UNED-MURCIA Universidad de las Islas Baleares.

Bruce, C. S. (2003). Las siete caras de la alfabetización en información en la enseñanza superior. Anales de Documentación, 6, 289-294. Extraído el 10 de abril, 2008 de

http://www.um.es/ojs/index.php/ analesdoc/article/view/3761/3661
Cabero, J. [Coord.]. (1994). Los medios audiovisuales y las nuevas tecnologías en la enseñanza de la lengua. En de las Heras, J. y otros (Eds.), IV Congreso sobre enseñanza de la lengua en Andalucía, (1-8). Huelva, Diputación Provincial de Huelva. Extraído el 2 de marzo del 2007 de http://edutec.rediris.es/documentos/1996/7.htm

Cabero, J. (2002). La aplicación de las TIC: ¿esnobismo o necesidad educativa? Revista de Tecnologías de la Información y Comunicación Educativas. Red Digital, 1. Extraído el 7 de abril de 2007 de

http://reddigital.cnice.mecd.es/1/ firmas/firmas_cabero_ind.html

Cabrera, P. (s.f.). Los cursos en línea de red escolar. Extraído el 12 de junio de 2007 de http://www.bibliotecadigital. conevyt.org.mx/colecciones/ documentos/somece/97.pdf

Chiecher, A., Donato, D. y Rinaudo, M. C. (2006, Julio-Agosto). Aprender en contextos virtuales por opción u obligación. Posibilidad de elección y perfiles cognitivos de alumnos universitarios. Revista Cognición, 6, 10-24. Extraído el 12 de octubre de 2007 de http://www.cognicion.net/cognicion/ files/anachiecheraprender contextos_virtuales.pdf

Council of Australian University Librarians. (2002). Normas sobre alfabetización en información. Boletín de la Asociación Andaluza de Bibliotecarios, 68, pp.67-90. Extraído el 20, julio de, 2007 de http://www. aab.es/pdfs/baab68/68a4.pdf

Dibut, L., Valdés G., Arteaga H., Toledo L., Toledo V. y Agustín S. (2006): Las nuevas tecnologías de la información y la comunicación como mediadoras 
del proceso de enseñanza-aprendizaje, (s.p.). Extraído el 2 de febrero del 2007 desde http://tecnologiaedu. us.es/edutec/paginas/61.html

Fainholc, B. (2004). El concepto de mediación en la tecnología educativa apropiada y crítica. Educar. Extraído el 8 de mayo del 2007 de

http://www.ftp.ihmc.us/servlet/SBRead ResourceServlet?rid=1119466861556_ $\underline{1804172076 \_502}$

Fazeli, H., Manzano, V. y Pérez, F. (2000). Variables contextuales en el éxito de las aplicaciones multimedia en la Universidad. Revista Electrónica de Metodología Aplicada, 1(5), 1-9. Extraído el 12 de mayo del 2007 de http://www.psico.uniovi.es/REMA/ v5n1/a1/

González, F. \& Salmon, G. (s.f.). La función y formación del E-moderator: Clave del éxito en los nuevos entornos de aprendizaje. Extraído el 15 de noviembre del 2006 desde

http://www.atimod.com/research/ presentations/educaspanish

Josemaría, C. (2001, Abril). Diseño de multimedias educativas. Libros en Red.com. Estudios Interdisciplinarios, Argentina. Extraído el 22 de abril del 2007 de http:// www.bibliotecas.reduaz.mx/libros-e/ libros/Claudio_Josemaria_AltisenDisenos_Multimedias_Educativos.pdf

López, M. C., Espinoza de los Monteros, A. y Flores, K. (2006). Percepción sobre las tecnologías de la información en los docentes de una universidad mexicana: el Centro Universitario del Sur de la Universidad de Guadalajara. Revista Electrónica de Investigación Educativa, 1(8) s.p. Extraído el 12 de setiembre del 2006 de http://redie.uabc.mx/vol8no1/ contenido-espinoza.html
Marquès, P. (2000). Los medios didácticos. Departamento de Pedagogía aplicada, Facultad de Educación UAB. Extraído el 12 de julio del 2007 desde http://dewey.uab.es/pmarques/medios. htm\#tipolog\%EDa

Marquès, P. (2001): Selección de materiales didácticos y diseño de intervenciones educativas. Extraído el 2 de marzo del 2007 desde http://www.dewey.uab.es/ pmarques/orienta.htm

Martínez, R. D., Montero, Y. H. y Pedrosa, M. E. (2001). La computadora y las actividades del aula: algunas perspectivas en la educación general básica de la provincia de Buenos Aires. Revista Electrónica de Investigación Educativa, 2(3). Extraído el 12 de mayo del 2007 desde

http://dialnet.unirioja.es/servlet/ $\underline{\text { articulo?codigo }=243651}$

Martínez, R. D., Montero, Y. H. y Pedrosa, M. E. (2005). La integración de la computadora a un ambiente de enseñanza y aprendizaje. Revista Iberoamericana de Educación, 35(1), (s.p). Extraído el 15 de junio de 2007 desde

http://www.rieoei.org/experiencias85. $\underline{\text { htm }}$

Martínez, R. D., Montero, Y. H., Pedrosa, M. E. y Martín, E. I. (2006). La capacitación docente en informática y su transferencia al aula: Un estudio en la provincia de Buenos Aires. Revista Electrónica de Investigación Educativa, 2(8). Extraído el 6 de noviembre del 2006 desde

http://redie.uabc.mx/vol8no2/ contenido-vidal2.html

Matas, A., Tójar, J. C. y Serrano, J. (2004). Innovación educativa: un estudio de los cambios diferenciales entre el profesorado de la Universidad de Málaga. 
Revista Electrónica de Investigación Educativa, 1(6), 1-21. Extraído el 10 de mayo del 2007 desde http://redie.uabc. $\mathrm{mx} /$ vol6no1/contenido-contenido.html

Onrubia, J. (2005, Febrero). Aprender y enseñar en entornos virtuales: actividad conjunta, ayuda pedagógica y construcción del conocimiento. Revista de Educación a Distancia, número monográfico II, 2-16. Extraído el 9 de febrero de 2006 de http://www.um.es/ ead/red/M2/

Salinas, J. (1999, febrero). Enseñanza flexible, aprendizaje abierto. Las redes como herramientas para la formación. Edutec: Revista Electrónica de Tecnología Educatia, 10, s.p. Extraído el 14 de marzo del 2007 de http://www.uib.es/depart/gte/ edutec-e/revelec10/revelec10.html

Silvera, C. (2005). La alfabetización digital: una herramienta para alcanzar el desarrollo y la equidad en los países de América Latina y el Caribe. Acimed, 13(1). Extraído el 10 de abril de 2006 de http://www. bvs.sld.cu/revistas/aci/vol13_1_05/ aci04105.htm

Sylverter, H. (2007). Gestión de la calidad a través de la cooperación. En G. Alfaro (Comp.). Promover la Universidad. Cinco Estrategias y un Dilema (pp 211-217). San José, Costa Rica: Universidad Estatal a Distancia. 
